CAHIERS DE

NARRATOLOGIE

\section{Cahiers de Narratologie}

Analyse et théorie narratives

$14 \mid 2008$

Prose d'idées : formes et savoirs

\title{
Avant-propos. Prose d'idées, prose de pensée, un bilan
}

Prose d'idées, prose de pensée, bilan d'une réflexion

Marie-Laure Acquier

\section{(2) OpenEdition}

\section{Journals}

Édition électronique

URL : http://journals.openedition.org/narratologie/644

DOI : 10.4000/narratologie.644

ISSN : 1765-307X

Éditeur

LIRCES

Référence électronique

Marie-Laure Acquier, « Avant-propos. Prose d'idées, prose de pensée, un bilan », Cahiers de Narratologie [En ligne], 14 | 2008, mis en ligne le 20 juillet 2010, consulté le 30 avril 2019. URL : http://

journals.openedition.org/narratologie/644; DOI : 10.4000/narratologie.644

Ce document a été généré automatiquement le 30 avril 2019

\section{c) $(1) \Theta$}

Cahiers de Narratologie - Analyse et théorie narratives est mis à disposition selon les termes de la licence Creative Commons Attribution - Pas d'Utilisation Commerciale - Pas de Modification 4.0 International. 


\title{
Avant-propos. Prose d'idées, prose de pensée, un bilan
}

\author{
Prose d'idées, prose de pensée, bilan d'une réflexion
}

\author{
Marie-Laure Acquier
}

1 Le numéro 14 des Cahiers de narratologie présente les travaux effectués en 2006 et 2007 par le groupe interdisciplinaire " Textes et prose d'idées », créé en 2005 au sein du CIRCPLESEA 3159. Ce groupe rassemble des spécialistes de littérature, de civilisation, dans les domaines de l'Espagne moderne, de l'Italie et de la France contemporaines. Il se propose d'étudier les textes relevant de la prose d'idées inscrite dans un cadre chronologique, géographique et culturel ouvert.

2 Les premiers travaux du groupe ont consisté à définir et à cerner un objet d'étude. La réflexion menée lors de la journée d'études intitulée : "prose d'idées : formes et savoirs " organisée par le groupe le 6 octobre 2006 s'est prolongée par des séances de séminaire au cours de l'année 2007. Ce numéro publie le texte des communications prononcées lors de la journée ainsi que la plupart des textes des intervenants invités dans le cadre du séminaire.

3 Ce numéro est donc consacré à la catégorie de la prose d'idées comme lieu d'interaction entre le champ du littéraire, les discours de savoir et les disciplines qui s'y rapportent. L'ensemble des études ici proposées conduit à poser la prose d'idées comme un objet dont toute l'étendue ne peut s'offrir que dans une analyse contrastée, fruit du croisement de compétences multiples. Leur approche d'ensemble, interdisciplinaire, réfléchit à l'intérêt de la prose d'idées pour l'histoire des formes dans sa combinaison avec l'épistémologie des disciplines. C'est pourquoi les contributeurs à ce numéro posent des questions d'ordre méthodologique, taxinomique, épistémologique en liaison avec des problèmes génériques, esthétiques, et plus généralement culturels qui sont tous nécessairement marqués par leur historicité.

4 Dans les études littéraires portant sur la période classique, la prose d'idées est abordée comme une catégorie textuelle qui rassemble une grande quantité de productions. Son polymorphisme générique n'a d'égal que sa variété thématique. Elle comprend des textes qui se situent généralement à la marge des grands genres canoniques (essais, discours, 
dialogues, entretiens, lettres, selon la classification offerte récemment par Stéphane Pujol pour le $\mathrm{XVIII}^{\mathrm{e}}$ siècle français $\left.{ }^{1}\right)$. D'un point de vue thématique, les œuvres répertoriées dans les chapitres consacrés à la prose d'idées dans les ouvrages traitant de littérature classique appartiennent le plus souvent à des sous-groupes de la littérature. Leurs dénominations a recours au terme englobant de "littérature ", au sens large d'écrit, assorti d'adjectifs sur lesquels repose le critère classificateur. Ces adjectifs relèvent souvent d'une discipline mais ce n'est pas toujours le cas: "littérature politique", " littérature morale ", "littérature nobiliaire ", « littérature scientifique », « littérature économique ", «littérature philosophique ", « littérature religieuse », etc. La diversité, voire l'éclatement thématique dont témoigne la variété de l'adjectivation trouve un contrepoids dans l'invariabilité du substantif. Ce type de classification thématique, fondée ou non, justifie l'emploi de l'expression « littérature d'idées » que Stéphane Pujol donne comme équivalente de celle de prose d'idées (une équivalence discutée par Jean-Louis Brau dans sa contribution sur la littérature picaresque). Se profile ainsi une prose d'idées aux thématiques les plus diverses, dont la bibliographie a tendance à s'occuper en fonction des frontières disciplinaires. Or, notre démarche se fonde au contraire sur la conviction que c'est précisément dans un dialogue interdisciplinaire que l'étude de la prose d'idées livre toute sa richesse.

5 Si le terme de littérature est à prendre dans cette série d'expression comme un synonyme d'écrit, il n'en reste pas moins que les textes de l'âge classique qui relèvent de ces sousgenres de la littérature sont -pour reprendre en partie la classification genettienne de Fiction et diction- tantôt non littéraires (corpus étudiés par Marc Marti), tantôt conditionnellement littéraires (les œuvres étudiées par Marie-laure Acquier) ou encore constitutivement littéraires (le roman picaresque étudié par Jean-Louis Brau). Ce qui tendrait à prouver que, dans l'analyse de la prose d'idées en tant que catégorie constituée, la littérarité est un critère parmi d'autres et qu'un des questionnements auxquels elle peut être soumise serait plutôt : quel est le travail de la littérature dans la prose d'idées? Comment donc étudier des textes qui valent davantage pour ce qu'ils disent que pour ce qu'ils sont (thématique vs formel ou rhématique chez Genette)?

6 Ce questionnement est complété par un autre axe de notre réflexion qui met en jeu la prose d'idées non comme catégorie textuelle constituée de l'âge classique mais comme appellatif mobilisable pour certains textes contemporains échappant également à toute classification générique canonique. Cette réflexion se situe dans le sillage de la démarche généalogique qu'accomplit Marielle Macé dans son ouvrage, Le temps de l'essai ${ }^{2}$. Elle y établit un lien entre une période, celle de Montaigne, où l'essai est prose d'idées au premier sens de l'expression que nous avons envisagé, et la période contemporaine, qui se réapproprie l'essai. Cette démarche généalogique autorise un autre type de questionnement et incite à réfléchir sur des textes tout aussi inclassables que l'essai, et susceptibles d'être rattachés à la prose d'idées. La prose d'idées ne fonctionne plus alors comme catégorie constituée, mais s'offre à une définition extensive. Restreindre la prose d'idées à la production des $\mathrm{XVII}^{\mathrm{e}}$ et $\mathrm{XVIII}^{\mathrm{e}}$ siècles reviendrait à la penser comme une catégorie morte, comme Gérard Genette parle de « genre mort » à propos du sonnet ou de l'épopée ${ }^{3}$. Une autre question devient alors pertinente : quels sont les textes qui peuvent relever de la prose d'idées? Ce renouvellement induit de la notion a conduit certains auteurs à revisiter des catégories textuelles inclassables : la littérature médicale (Edwige Fusaro), les manifestes esthétiques (Serge Milan), la littérature des camps (Lucie Bertrand), afin d'éprouver de façon novatrice la fonction définitoire de la prose d'idées. 
Un cas limite étant celui de la " prose culinaire » étudiée par Cécile de Bary. Comme dans un mouvement dialectique et rétrospectif, cette extension de la catégorie enrichit l'analyse de certaines productions textuelles de l'ancien régime et permet à Jean-Louis Brau d'envisager l'archétype du roman picaresque espagnol et à Anne Coudreuse de penser l'écriture de soi à l'œuvre dans les Mémoires de Marmontel comme de la prose d'idées.

7 Cet angle d'attaque redonne toute son expressivité à un appellatif parfois décrié et restreint à un usage académique et qui, à bien y regarder, forme un tout cohérent. Dans l'expression "prose d'idées", la prose comme catégorie ordonnatrice est en soi un élément médiat comme l'idée en est un autre en tant qu'il médiatise la relation entre l'objet et l'esprit et ressortit à l'enjeu de la connaissance. L'élément «prose » renvoie à une forme de discours, agit comme élément général classificateur, définit une catégorie englobante qui transcende les genres. En d'autres termes, si les problématiques liées à la prose d'idées dépassent la question générique, elles ne l'oblitèrent pas. Les médiations génériques sont à l'œuvre dans la prose d'idées. Elles permettent de lui appliquer un questionnement de type littéraire sans pour autant en interdire un autre, qu'amorce déjà Marielle Macé à propos du genre de l'essai. Dans sa contribution à ce numéro, Marielle Macé se demande quel type de savoir dispense l'essai afin d'évaluer un moment culturel qui contraint la littérature à se positionner par rapport aux discours de savoir. Par un double mouvement de généralisation et décentrage, se posent les questions suivantes : quels sont les savoirs mis en jeu par la prose d'idées? Selon quelles modalités la prose d'idées met-elle en jeu ces savoirs? Avec quels enjeux pour l'existence même de la prose d'idées en tant que catégorie textuelle ? Il est permis alors d'envisager la définition de la prose d'idées en termes d'appropriation. En quoi l'appropriation de la prose d'idées par la littérature ou par d'autres champs disciplinaires en invertit la définition et à quel moment?

\section{Un « empire du milieu », la question de la fiction}

Pour la prose d'idées, le lien à la littérature, s'il est fondamental et déterminant, est très complexe et ambigu. Une des façons de l'envisager se fonde sur les mécanismes de mise en œuvre de la fiction dans la prose d'idées. Un grand nombre des textes étudiés dans ce volume relèvent à première vue d'une prose non fictionnelle: la "littérature " économique, éthique et politique (corpus de Marc Marti), les mémoires (Anne Coudreuse), le manifeste esthétique (Serge Milan), l'essai (Marielle Macé), l'écriture des camps (Lucie Bertrand), la prose culinaire (Cécile de Bary). Ils sont parfois tout imprégnés du déni de fiction propre au témoignage (Mémoires, expérience des camps), qui traduit une grande défiance à l'égard de la littérature perçue comme un artifice face aux grands problèmes historiques de notre temps. Le point de départ de l'analyse de Lucie Bertrand sur l'œuvre de Robert Antelme est bien une impossible "littérature des camps ». D'autres types de textes étudiés jouent de la fiction propre à un genre, comme le songe (Marie-Laure Acquier), ou le roman (Jean-Louis Brau, Edwige Fusaro). Pourtant, ces textes, si divers soient-ils chronologiquement et génériquement, se rejoignent dans la façon parfois ambiguë et retorse dont ils mettent en œuvre la fiction et ses recours.

Dans le cas des récits romanesques, qu'il s'agisse du Guzmán de Alfarache de Mateo Alemán revisité par Jean-Louis Brau et Un Jour à Madère de Paolo Mantegazza étudié par Edwige Fusaro, la fiction ne vaut jamais pour elle-même mais parce qu'elle ouvre la voie à 
l'exemplarité. L'archétype du roman picaresque est régi comme toute production littéraire de la littérature européenne de l'âge classique par le prodesse horacien où émouvoir et convaincre, où plaisir et utilité sont indissociables. Néanmoins, dans le cas du Guzmán, le dosage entre fiction et morale est proportionnel au volume occupé dans l'économie de l'œuvre par les sermons intercalés. Leur poids éthique, en rapport constant avec l'apprentissage du personnage fictionnel du gueux, est tel qu'il conduit à évaluer le roman comme un exemplum démesuré illustrant un sermon non moins démesuré. Ill pose de manière induite la question théologique de l'ornementation dans l'art oratoire. Chez Paolo Mantegazza, dans un contexte bien différent, celui de l'apogée des élites scientifiques dans l'Italie post-unitaire, la matière romanesque n'est que le support expérimental d'un programme sanitaire et hygiéniste (éviter la propagation de la tuberculose), à son tour vecteur d'une morale eugénique. Dans les deux cas, l'instrumentalisation de la fiction est combinée à un hybridation générique, où le sermon prend le pas sur le roman, où le récit romanesque oscille entre roman épistolaire, récit de voyage, littérature édifiante et traité de vulgarisation médicale. Cette mise en œuvre de la fiction est également marquée par un phénomène publicitaire, le succès de la narration en prose dans l'Espagne de la fin du XVI ${ }^{\mathrm{e}}$ siècle dans un cas, l'immense retentissement des œuvres romanesques dans la France et l'Italie de la fin du XIX siècle dans l'autre. Le constat est similaire pour le cas du songe d'Antonio López de Vega daté de la première moitié du XVII ${ }^{e}$ siècle espagnol (Marie-Laure Acquier). Dès le XVII siècle, en Espagne, un manuel d'éducation du noble se coule sous les traits séducteurs d'un récit onirique investissant le genre du songe et empruntant l'énonciation de l'autobiographie fictive, celle du roman picaresque en vogue. Le récit d'un modeste serviteur transporté oniriquement au pied de la demeure somptueuse de la Philosophie pourra ainsi dessiner le modèle du parfait courtisan, dans le dialogue fictif qui s'établit entre les deux personnages et qui a tous les traits du dialogue humaniste. Dans ces trois cas, la prose d'idées suppose l'hybridation générique, support de la variété thématique qui lui est propre et lié au polygraphisme des auteurs.

10 La production des Lumières espagnoles étudiée par Marc Marti, cas exemplaire de ce polygraphisme, a généré des errements notoires dans la catégorisation de ces textes. Comme le démontre Marc Marti, ces hésitations sont autant de marqueurs de l'enjeu épistémologique de l'investissement de ces textes par l'une ou l'autre discipline, entre littérature et histoire des idées. Jusqu'au risque du paradoxe, les Mémoires de JeanFrançois Marmontel qui relèvent pourtant d'un genre considéré comme non fictionnel entretient des rapports complexes avec la fiction. Anne Coudreuse démonte le mécanisme d'une écriture de soi qui puise dans les modèles fictifs de l'époque pour construire une écriture du pathétique. Les pleurs de Marmontel entrent dans la stratégie d'autojusfication de sa palinodie. Celle-ci contraint le récit à des rapports ambigus avec la vérité des faits pour éclairer les deux visions contradictoires que l'auteur nous offre, de la pensée des Lumières qu'il défend et de l'action révolutionnaire qu'il réprouve.

11 La fiction paraît alors dispenser cette sorte d'exemplarité dont Raphael Baroni disait, dans un récent entretien accordé à Franck Wagner dans la revue vox poetica ${ }^{4}$, qu'elle est « non pas d'ordre conceptuel mais sensible » et qu'elle « rend transférable la valeur d'une série d'événements irréductiblement singuliers ». C'est sans nul doute sa malléabilité et son caractère « transférable » qui rend la fiction aussi propice à réappropriation dans les textes de prose d'idées. Elle peut ainsi rejoindre « les cas mémorables ou remobilisables » 
qui sont, selon Marielle Macé, tout le savoir dispensé par l'essai, considéré comme genre non fictionnel par excellence.

Les récentes études littéraires sur l'effet de fiction ${ }^{5}$ montrent qu'un "désir de fiction " affecte toute production littéraire et la tire vers une scénarisation de l'énoncé qui tout aussi bien permet une accroche de la lecture, procure les affects liés à la tension narrative sans pour autant recourir à une construction imaginaire fictionnelle conçue selon les représentations classiques. Les manifestes italiens de l'avant-garde futuriste étudiés par Serge Milan qui proposent dans un style incisif et provocateur une société nouvelle, scénarisent en se projetant dans l'avenir l'avènement de la violence et un enchaînement de faits apte à déboucher sur une humanité régénérée. Le projet social, humain, philosophique du manifeste se met en récit tout en fonctionnant sur le contre-modèle d'une utopie destructrice mais régénératrice qui fait appel aux ressorts de l'imaginaire et s'apparente à la fiction. Nous sommes là au point de rencontre entre la littérature, l'art et la philosophie. La prose d'idées pourrait alors envahir cet «empire du milieu » que décrivait Gérard Genette entre fiction et diction où les notions de porosité, d'interaction, d'hybridation, d'« interstices » (terme dû à Raphaël Baroni) finissent par rendre floue celle de frontière.

\section{L'idée et la voix}

Ces liens ambigus et retors à la fiction, dont découlent les problèmes taxinomiques et génériques déjà relevés dans la prose d'idées, dépendent en grande partie de sa caractérisation comme lieu de réflexion et d'élaboration intellectuelle. L'exemplarité de la fiction dans la prose d'idées se met le plus souvent au service d'un «discours spéculatif » et/ou d'une "rhétorique de type argumentatif », pour reprendre des termes dus à Stéphane Pujol dans la définition qu'il donne de la prose d'idées au XVIII ${ }^{\mathrm{e}}$ siècle. La prose d'idées développe un argumentaire implicite ou explicite et entend convaincre ou enseigner. Elle modèle son lectorat. Toutes les productions apparentées à l'archigenre de la conservation, mais aussi toutes celles qui obéissent au schéma discursif du protagoniste et de l'antagoniste contribuent dans leurs modalités d'écriture à l'émergence du débat d'idées au sein du texte. On retrouvera dans l'ensemble des contributions à ce numéro l'une ou l'autre de ces dimensions de la prose d'idées qui prend alors dans certains cas des allures polémiques voire militantes ou provocatrices.

Pour la période classique, l'ouverture au débat d'idées dans et par la prose d'idées est fondamentale et ses implications connues. L'émergence de nouveaux publics incite à la diffusion des savoirs grâce aux apports de la pédagogie humaniste qui ouvre la voie au didactisme. Les nouvelles conditions de production du livre, la préoccupation pour la réception qu'elles engendrent et supposent (souvent lisible dans les paratextes), suscitent, avec et malgré les freins et les réticences de la censure, l'ouverture du champ public au débat d'idées. La pensée des Lumières qui s'élabore dans le laboratoire de la prose d'idées étudiée par Marc Marti atteste cette évolution. La contamination inattendue de rapports administratifs et de projets de réforme économique par une pensée et une rhétorique du bonheur dans l'Espagne du XVIII ${ }^{\mathrm{e}}$ siècle traduit une généralisation du débat d'idées jusqu'à son institutionnalisation; une façon pour les autorités de reprendre en main ce qui, comme dans le cas français, aurait pu un moment leur échapper. La portée idéologique de la prose d'idées peut alors se mesurer dans les stratégies d'écriture. A cet égard, il est intéressant de comprendre avec Anne Coudreuse comment cette écriture de 
soi et de l'épanchement que sont les Mémoires de Marmontel travaille dans le sens d'une justification du positionnement idéologique de l'auteur. Par la construction du récit, Marmontel récupère l'efficacité dialogique à l'œuvre dans la prose d'idées tout en respectant l'énonciation du «je » narrant caractéristique du genre. Ce dédoublement ambigu de la voix permet à Marmontel de proposer deux jugements contradictoires sur la Révolution à travers la même voix. Il offre la mémoire d'une vie et une mémoire de la Révolution.

A la recherche du naturel, de l'authenticité ou d'une force de conviction et d'engagement, les textes de prose d'idées ont une prédilection pour l'énonciation à la première personne. Qu'elle soit propre à un genre comme l'autobiographie ou l'essai (Jean-Louis Brau, Marielle Macé), à une modalité de discours comme le dialogue ou le témoignage (Marie-Laure Acquier, Lucie Bertrand), son compagnonnage avec la prose d'idées s'installe dans la durée au service d'une volonté de transmettre des idées et comme vecteur d'idéologie ou d'une pensée. La prose passéiste de Claudian (Cécile de Bary), son lyrisme daté, sa volonté d'archiver un savoir-faire conjugués à l'irruption du « je » dans la recette proclament son idéologie réactionnaire dans une période où les décolonisations perturbent les certitudes établies de l'européocentrisme. L'étude par Marielle Macé du phrasé de l'essai et de sa prise en charge par son énonciation spécifique saisit une façon d'imposer des jugements péremptoires, des "raccourcis de pensée ", des "précipités d'idées », cette chimie verbale portée par un « je » qui suscite d'emblée l'approbation du lecteur ou son irritation. Jusqu'à ce considérable effort de Robert Antelme pour effacer la portée polémique de la notion de littérature des camps et rendre compatible les deux termes d'un oxymore : dire l'indicible; Lucie Bertrand démonte les mécanismes de cette inévitable littérarisation de l'expérience vécue qui tente de se défaire de tout ornement pour atteindre le cru et le nu, et porter à travers le récit d'un « je » qui se veut collectif l'universalité d'un message: celui du plus jamais ça. Le "je» collectif de l'écriture des camps telle que la pratique Robert Antelme, sa volonté d'accéder à l'universel à travers l'expérience particulière d'un homme qui doit transmettre à tous les hommes, s'apparente au sujet philosophique en cela qu'il tend au général à partir de l'expérience particulière. Il rejoint à cet égard le «nous » des manifestes futuristes dont l'idéologie de la violence ancrée dans le temps de la guerre et dans l'action de la grève s'élève à l'universalité philosophique dans une théorie totalisante et auto-référentielle propre aux avant-gardes (Serge Milan).

\section{L'enjeu des savoirs, la question du temps}

Les contributions à ce numéro offrent une palette très ouverte des disciplines mobilisées et des savoirs produits dans et par la prose d'idées : éthique, économie, politique (JeanLouis Brau, Marie-Laure Acquier, Marc Marti), histoire (Anne Coudreuse, Lucie Bertrand), médecine (Edwige Fusaro), art et philosophie (Serge Milan). L'observation, l'apprentissage, l'expérience, la mémoire, les savoir-faire investissent les formes du récit ou du discours qui en valorisent la captation par le lecteur. Ils s'offrent à l'interprétation comme autant de connaissances sur le champ de la pensée en un temps donné (Marc Marti). Par voie de conséquences, la prose d'idées, ses savoirs et leur « mise en œuvre » - à la fois comme mise en pratique et comme mise en composition littéraire - sont affectés par l'historicité propre aux champs disciplinaires dont les contours sont très variables dans le temps. Le positionnement de la littérature par rapport aux sciences humaines - 
dont Marielle Macé étudie un moment à travers le réinvestissement des discours de savoir par l'essai- est à envisager dans un mouvement dialectique. Les études historiques et littéraires se sont donné comme objet d'étude l'histoire des processus de "littérarisation" des productions intellectuelles; de la même façon, l'histoire des disciplines concernées par cette « littérarisation » se donne à comprendre en regard de l'histoire de la littérature comme discipline.

Dans une époque -celle des temps modernes- où les lettres embrassent tous les domaines de la connaissance et des savoirs, la circulation des concepts sous une forme textuelle donnée apporte de l'information sur leur audience et leur prégnance, sur les fluctuations épistémologiques qui les affectent en relation avec l'émergence ou les interactions des champs disciplinaires. Les auteurs de prose d'idées y revendiquent leur volonté de dispenser un savoir utile en publiant leurs ouvrages par voie manuscrite ou imprimée. Cette conscience de la diffusion du savoir confère toute son importance aux connaissances qu'ils communiquent, d'abord pour ce qu'elles sont, mais aussi par leur rattachement à un domaine donné du savoir. Ce rattachement est souvent opéré par les auteurs eux-mêmes dans les pièces paratextuelles. L'exemple des trois modes de gouvernements éthique, économique, politique qui revient à deux reprises dans les contributions à ce numéro (Marie-Laure Acquier, Marc Marti) est significatif à cet égard, dans son rapport avec l'histoire des formes et par ses implications épistémologiques. L'étude de ces trois modes dans une forme donnée (littéraire ou non littéraire) permet de mesurer les avancées ou les réticences des représentations collectives par rapport à la constitution des disciplines. Elle informe sur l'histoire de la norme et du consensus social et sur l'histoire des écarts par rapport à un modèle précis.

Cette écriture de l'utilité, ses implications épistémologiques en un temps historique donné, l'investissement littéraire de la connaissance ou du champ de la pensée disent beaucoup sur le positionnement de la littérature par rapport aux discours de savoir. C'est dans cette perspective que Marielle Macé replace l'histoire du genre de l'essai et de sa réapparition sur la scène littéraire au $\mathrm{XX}^{\mathrm{e}}$ siècle. Tout en refusant au genre de l'essai en lui-même des implications épistémologiques en ce que l'on y voit la pensée à l'œuvre et non pas le savoir, Marielle Macé saisit son investissement par la tradition littéraire française comme un moment culturel, celui de la concurrence entre disciplines, entre la littérature et les sciences humaines. L'essai, ce laboratoire de la Topique dans un style du prêt-à-citer (mais les lieux communs ne sont-ils pas une sorte de savoir renseignant sur une époque?), met en regard un «moment agonique» des genres littéraires et une «querelle de propriété » disciplinaire. Marielle Macé y scrute cette écriture de l'utile dans le « mémorable » et le « citable » qui entend s'approprier -mais dénote- la culture en un temps donné. Cette inscription dans le temps d'une écriture par la cristallisation d'un moment culturel est aussi une des modalités de l'historicité propre à toute production textuelle, à saisir par sa préalable contextualisation.

19 La prose d'idées est encore affectée par une autre sorte de temporalité propre au type de savoir utile qu'elle entend dispenser. Réserve d'expériences, celle d'un gueux repenti (Jean-Louis Brau) ou d'une tuberculeuse (Edwige Fusaro), magasin de modèles -le parfait courtisan (Marie- Laure Acquier) ou le père vertueux (Anne Coudreuse )- fabrique de lieux communs (Marielle Macé), elle produit des normes (Marie-Laure Acquier, Marc Marti) et affirme des valeurs (l'idéologie réactionnaire de Claudian, mise en lumière par Cécile de Bary ou celle de l'avant-garde futuriste qu'analyse Serge Milan). Elle s'adresse en cela à l'homme en tant qu'être social, en tant qu'être dans le temps. Qu'il s'assigne une visée 
rétrospective (mémoire, histoire comprise dans ses deux acceptions -fable ou récit d'événements historiques-) et/ou une visée prospective (projets de réforme, apport scientifique, idéologie du progrès), ce savoir utile à l'homme et sur l'homme est en quête de général à partir de singularités. Cette ouverture à l'appropriation par tous les hommes, cette volonté d'atteindre l'universel par la philosophie ou l'éthique (Jean-Louis Brau, Lucie Bertrand, Serge Milan) créent une tension particulière dans la prose d'idées puisqu'elle est irrémédiablement datée. Cette discordance liée à un ancrage dans le temps à plusieurs échelles (savoirs et implications disciplinaires, contextualisation et appropriation-réception-interprétation), et qui produit parfois de l'anachronisme, ne freine pourtant pas la capacité englobante de la catégorie. Sa fonction extensive reste encore largement à explorer.

Affectée par l'hybridation générique, les interactions entre champs disciplinaires, la prose d'idées apparaît comme une catégorie de l'impur. Par nature, elle résiste à tout étiquetage. Parce qu'elle peut être tout autant un objet littéraire qu'un objet historique, philosophique, ou scientifique, elle perturbe les classifications instituées et oblige à une remise à plat des cloisonnements disciplinaires. La question des usages de la catégorie a bien montré les errements de toute tentative classificatoire. L'analyse littéraire nous fournit des clés indispensables pour l'appréhender mais toujours à combiner avec d'autres compétences. Son mérite réside en ce qu'elle contraint à créer de la relation pour être comprise. Elle reste néanmoins elle-même sujette à une tension particulière entre un désir de permanence et de général et l'ancrage dans un moment culturel qui découle des paradoxes liés à son historicité. C'est dans une narrativité propre alliant éléments fictionnels et factuels qu'elle peut être saisie dans toute sa richesse.

\section{NOTES}

1. Michel Prigent, Histoire de la France littéraire, tome 2, Classicismes. XVIIe-XVIIIe siècle, JeanCharles Darmon, Michel Delon (dir.), Paris, Presses Universitaires de France, 2006, p. 701- 731.

2. Paris, Gallimard, 2006.

3. Fiction et diction, Paris, Seuil, 1991, p. 30

4. http://www.vox-poetica.org/entretiens/baroni.html, entretien publié le 03/07/2007, page consultée le 24 janvier 2008.

5. Les colloques en ligne "frontières de la fiction" (1999), "l'effet de fiction" (2001) explorent ces thèmes sur le site de l'équipe de recherche Fabula. Adresses respectives http://www.fabula.org/ forum/colloque99.php et http://www.fabula.org/effet/. Pages consultées le 25 février 2008. 


\section{AUTEUR}

\section{MARIE-LAURE ACQUIER}

Université de Nice-Sophia Antipolis, CIRCPLES-EA 3159

Spécialiste de la prose d'idées espagnole des XVIe et XVIIe siècles, Marie-Laure Acquier est responsable du groupe de recherche " prose d'idées, littératures d'idées » au sein du CIRCPLES-

EA 3159. 